\title{
A Linear Bound towards the Traceability Conjecture
}

\author{
Susan A. van Aardt* \\ Department of Mathematical Sciences \\ University of South Africa \\ Gauteng, South Africa \\ vaardsa@unisa.ac.za \\ Marietjie Frick ${ }^{\dagger}$ \\ Department of Mathematical Sciences \\ University of South Africa \\ Gauteng, South Africa \\ marietjie.frick@gmail.com
}

\author{
Jean E. Dunbar \\ Department of Mathematics \\ Converse College \\ South Carolina, USA \\ jean.dunbar@converse.edu \\ Nicolas Lichiardopol \\ Lycee A. de Capronne \\ Salon \\ France \\ nicolas.lichiardopol@neuf.fr
}

Submitted: Oct 4, 2014; Accepted: Nov 3, 2015; Published: Nov 13, 2015

Mathematics Subject Classifications: 05C20, 05C38

\begin{abstract}
A digraph is $k$-traceable if its order is at least $k$ and each of its subdigraphs of order $k$ is traceable. An oriented graph is a digraph without 2-cycles. The 2 -traceable oriented graphs are exactly the nontrivial tournaments, so $k$-traceable oriented graphs may be regarded as generalized tournaments. It is well-known that all tournaments are traceable. We denote by $t(k)$ the smallest integer bigger than or equal to $k$ such that every $k$-traceable oriented graph of order at least $t(k)$ is traceable. The Traceability Conjecture states that $t(k) \leqslant 2 k-1$ for every $k \geqslant 2$ [van Aardt, Dunbar, Frick, Nielsen and Oellermann, A traceability conjecture for oriented graphs, Electron. J. Combin., 15(1):\#R150, 2008]. We show that for $k \geqslant 2$, every $k$-traceable oriented graph with independence number 2 and order at least $4 k-12$ is traceable. This is the last open case in giving an upper bound for $t(k)$ that is linear in $k$.
\end{abstract}

Keywords: Oriented graph, Generalized tournament, $k$-traceable, Traceability Conjecture, Path Partition Conjecture

*Supported by the National Research Foundation of S.A, Grant 81075.

†Supported by the National Research Foundation of S.A, Grant 81004. 


\section{Introduction and Background}

A digraph is hamiltonian if it contains a cycle that visits every vertex, traceable if it contains a path that visits every vertex.

A digraph is $k$-traceable if its order is at least $k$ and each of its subdigraphs of order $k$ is traceable. A digraph without 2-cycles is called an oriented graph. It is easily seen that an oriented graph is 2-traceable if and only if it is a nontrivial tournament. Thus $k$-traceable oriented graphs may be regarded as generalized tournaments. It is well-known that every nontrivial strong tournament is hamiltonian and every tournament is traceable. The following theorem, which follows from results in $[3,5,12]$, shows that these properties are retained by $k$-traceable oriented graphs for small values of $k$.

Theorem 1.1. [3, 5, 12]

1. For $k=2,3,4$, every strong $k$-traceable oriented graph of order at least $k+1$ is hamiltonian.

2. For $k=2,3,4,5,6$, every $k$-traceable oriented graph is traceable.

However, it is shown in [5] that for $k \geqslant 5$ there exists a nonhamiltonian strong $k$ traceable oriented graph of order $n$ for every $n \geqslant k$. Furthermore, it is shown in [7] that for $k=7$ and for every $k \geqslant 9$ there exist $k$-traceable oriented graphs of order $k+1$ that are nontraceable. (Such graphs are called hypotraceable). There also exist nontraceable $k$-traceable oriented graphs of order $k+2$ for infinitely many $k$, as shown in [6]. These observations lead naturally to the following question, posed in [3].

Question 1. For $k \geqslant 2$, what is the smallest integer $t(k)$ such that $t(k) \geqslant k$ and every $k$-traceable oriented graph of order at least $t(k)$ is traceable?

The Traceability Conjecture (or TC for short), which is studied in $[1,3,4,5,12]$ may be stated as follows.

Conjecture 1. (TC) $t(k) \leqslant 2 k-1$ for every $k \geqslant 2$.

As explained in [5], settling the TC could be an important step towards settling the Path Partition Conjecture for Digraphs. The latter conjecture was motivated by the paper [14] by Laborde, Payan and Xuong and is discussed in [2, 8, 9].

Theorem 1.1 and results in $[1,3,10]$ imply the following.

Theorem 1.2. $[1,3,10]$

$t(k)=k$ for $2 \leqslant k \leqslant 6$

$t(7)=9$

$t(8) \leqslant 14$

$t(k) \leqslant 2 k^{2}-20 k+59$ for every $k \geqslant 9$.

The TC motivated us to search for an upper bound for $t(k)$ that is linear in $k$. Van Aardt, Dunbar, Frick and Nielsen [5] proved the following result with respect to oriented graphs with independence number greater than 2 . 
Theorem 1.3. [5] If $k \geqslant 4$ and $D$ is a $k$-traceable oriented graph with $\alpha(D) \geqslant 3$ and $n(D) \geqslant 6 k-20$, then $D$ is traceable.

In this paper we show that for $k \geqslant 4$, every $k$-traceable oriented graph with independence number 2 and order at least $4 k-12$ is traceable. This then proves that $t(k) \leqslant 6 k-20$ for every $k \geqslant 4$ and thus brings us significantly closer to settling the TC.

\section{Notation and Auxilliary Results}

For undefined concepts we refer the reader to [8].

The set of vertices and the set of arcs of a digraph $D$ are denoted by $V(D)$ and $A(D)$, respectively, and the order of $D$ is denoted by $n(D)$. If $X \subset V(D)$, then $\langle X\rangle$ denotes the subdigraph induced by $X$ in $D$. The independence number of $D$, denoted by $\alpha(D)$, is the cardinality of a largest set $X \subset V(D)$ such that $\langle X\rangle$ has no arcs.

If $v \in V(D)$, we denote the sets of out-neighbours and in-neighbours of $v$ in $D$ by $N^{+}(v)$ and $N^{-}(v)$, respectively. The set $N(v)=N^{+}(v) \cup N^{-}(v)$ is simply called the neighbourhood of $v$. If $S$ is a subset of $V(D)$ or a subdigraph of $D$, we denote the set of neighbours, in-neighbours and out-neighbours of $v$ in $S$ by $N_{S}(v), N_{S}^{-}(v)$ and $N_{S}^{+}(v)$, respectively.

A digraph $D$ is strong (or strongly connected) if for every pair of distinct vertices $u, v$ in $D$ there is a path from $u$ to $v$. A maximal strong subdigraph of a digraph $D$ is called a strong component of $D$. The strong components of $D$ have an acyclic ordering $D_{1}, D_{2}, \ldots, D_{h}$ such that if there is an arc from $D_{i}$ to $D_{j}$, then $i \leqslant j$. If $D$ is $k$-traceable for some $k \geqslant 2$, this acyclic ordering is unique since there is at least one arc from $D_{i}$ to $D_{i+1}$ for $i=1,2, \ldots, h-1$. Throughout this paper we label the strong components of a $k$-traceable digraph in accordance with this acyclic ordering. We denote by $D_{r}^{s}$ the subdigraph of $D$ induced by the vertex set $\bigcup_{i=r}^{s} V\left(D_{i}\right)$.

Chen and Manalastas [11] proved that every strong digraph with independence number two is traceable. Havet [13] strengthened their result by proving that if $D$ is a strong digraph with $\alpha(D)=2$, then $D$ has two nonadjacent vertices that are terminal vertices of Hamilton paths in $D$ and two nonadjacent vertices that are initial vertices of Hamilton paths in $D$. The following theorem, which follows from Havet's result, is proved in [3].

Theorem 2.1. [3] If $D$ is a connected digraph with $\alpha(D)=2$ and at most two strong components, then $D$ is traceable.

We shall frequently use the following result.

Lemma 2.2. [1] Let $G$ be a $k$-traceable oriented graph of order $n$. Then the following hold.

1. $|N(x)| \geqslant n-k+1$ for every $x \in V(G)$.

2. $\left|N^{-}(x) \cup N^{-}(y)\right| \geqslant n-k+1$ and $\left|N^{+}(x) \cup N^{+}(y)\right| \geqslant n-k+1$ for every pair of nonadjacent vertices $x$ and $y$ in $G$. 
The following theorem follows from [1], Lemma 10 and Corollary 12.

Theorem 2.3. Let $k \geqslant 7$ and suppose $D$ is a nontraceable $k$-traceable oriented graph of order $n \geqslant 2 k-3$ with independence number 2. Let $D_{1}, \ldots, D_{h}$ be the strong components of $D$. Then $h \geqslant 3$ and there exists $a t \in\{2, \ldots, h-1\}$ such that $D_{t}$ is nonhamiltonian, while $D_{1}^{t-1}$ as well as $D_{t+1}^{h}$ are tournaments. Moreover, $n\left(D_{t}\right) \geqslant n-k+5$.

Next we state a lemma for the particular case $h=3$, which is used in our main theorem. It follows from results in $[1,3,5]$, but for ease of reference we provide a proof.

Lemma 2.4. Let $k \geqslant 7$ and suppose $D$ is a nontraceable $k$-traceable oriented graph of order $n \geqslant 2 k-3$ with independence number 2 and exactly three strong components $D_{1}, D_{2}, D_{3}$. Let $n\left(D_{i}\right)=n_{i}, i=1,2,3$. Then the following hold.

1. If $P$ is a Hamilton path in $D_{2}$ whose initial vertex has an in-neighbour in $D_{1}$, then the terminal vertex of $P$ does not have an out-neighbour in $D_{3}$.

2. $D_{2}$ is $\left(k-n_{1}-n_{3}\right)$-traceable.

3. $\left|N_{D_{2}}(x)\right| \geqslant n-k+1$ for every $x \in V\left(D_{2}\right)$.

4. If $x$ and $y$ are two nonadjacent vertices in $D_{2}$, then

(a) $\left|N_{D_{2}}^{+}(x) \cup N_{D_{2}}^{+}(y)\right| \geqslant n-k+1$,

(b) $\left|N_{D_{2}}^{-}(x) \cup N_{D_{2}}^{-}(y)\right| \geqslant n-k+1$.

5. (a) $\left|N_{D_{2}}^{+}\left(D_{1}\right)\right| \geqslant n-k+1$,

(b) $\left|N_{D_{2}}^{-}\left(D_{3}\right)\right| \geqslant n-k+1$.

6. (a) If $x \in V\left(D_{2}\right)$ and $x \notin N^{+}\left(D_{1}\right)$, then $\left|N_{D_{2}}^{-}(x)\right| \geqslant n-k+1$,

(b) If $x \in V\left(D_{2}\right)$ and $x \notin N^{-}\left(D_{3}\right)$, then $\left|N_{D_{2}}^{+}(x)\right| \geqslant n-k+1$.

Proof.

1. Suppose the initial vertex of $P$ has an in-neighbour $y$ in $D_{1}$ and the terminal vertex of $P$ has an out-neighbour $z$ in $D_{3}$. By Theorem 2.3, each of $D_{1}$ and $D_{3}$ is a strong tournament and hence is either hamiltonian or a single vertex. Thus $D_{1}$ has a path $Q$ with $y$ as terminal vertex, and $D_{3}$ has a path $R$ with $z$ as initial vertex. But then the path $Q P R$ is a Hamilton path of $D$, contradicting our assumption that $D$ is nontraceable.

2. From Theorem 2.3 and our assumption that $n \geqslant 2 k-3$ it follows that $0<k-$ $n_{1}-n_{3}<n_{2}$. Now consider any subdigraph $H$ of $D_{2}$ with $n(H)=k-n_{1}-n_{3}$. Let $H^{*}=\left\langle V(H) \cup V\left(D_{1}\right) \cup V\left(D_{3}\right)\right\rangle$. Then $n\left(H^{*}\right)=k$, so $H^{*}$ is traceable since $D$ is $k$-traceable. Let $P=v_{1} \ldots v_{k}$ be a Hamilton path of $H^{*}$. Then, due to the acyclic ordering of the strong components, the intersection of the path $P$ with the strong component $D_{2}$ is a Hamilton path of $H$. This proves that $D_{2}$ is $\left(k-n_{1}-n_{3}\right)$ traceable. 
3. It follows from (2) above and Lemma 2.2(1) that $\left|N_{D_{2}}(x)\right| \geqslant n_{2}-\left(k-n_{1}-n_{3}\right)+1=$ $n-k+1$.

4. This follows directly from (2) and Lemma 2.2(2).

5. If $\left|N_{D_{2}}^{+}\left(D_{1}\right)\right| \leqslant n-k$, then $\left|V\left(D_{2}\right)-N_{D_{2}}^{+}\left(D_{1}\right)\right| \geqslant n_{2}-(n-k)=k-n_{1}-n_{3}$, so we can choose a set $S \subseteq\left(V\left(D_{2}\right)-N_{D_{2}}^{+}\left(D_{1}\right)\right)$ such that $|S|=k-n_{1}-n_{3}$. Then the subdigraph $\left\langle V\left(D_{1}\right) \cup S \cup V\left(D_{3}\right)\right\rangle$ has order $k$ but is nontraceable, contradicting that $D$ is $k$-traceable. This proves $5(\mathrm{a})$. The proof of $5(\mathrm{~b})$ is similar.

6. If $\left|N_{D_{2}}^{-}(x)\right| \leqslant n-k$, then we choose a subset $S$ with $|S|=k-n_{1}-n_{3}$ such that $x \in S \subseteq\left(V\left(D_{2}\right)-N_{D_{2}}^{-}(x)\right)$. But then the subdigraph $\left\langle V\left(D_{1}\right) \cup S \cup V\left(D_{3}\right)\right\rangle$ has order $k$ but is nontraceable, since there are no arcs from $D_{1}$ to $S$. This proves $6(\mathrm{a})$. The proof of $6(\mathrm{~b})$ is similar.

\section{Main Result}

Theorem 3.1. Let $k \geqslant 2$ and suppose $D$ is a $k$-traceable oriented graph such that $\alpha(D)=$ 2 and $n(D) \geqslant 4 k-12$. Then $D$ is traceable.

Proof. The proof is by induction on $k$. By Theorem 1.2, the result holds for $k \leqslant 8$. Now let $k \geqslant 9$ and let $D$ be a $k$-traceable oriented graph with independence number 2 and order $n \geqslant 4 k-12$. Suppose $D$ is nontraceable and let $D_{1}, \ldots, D_{h}$ be the strong components of $D$, with $n\left(D_{i}\right)=n_{i}, i=1, \ldots, h$. Then, by Theorem $2.3, h \geqslant 3$ and $D$ has a nonhamiltonian strong component $D_{t}$ of order at least $n-k+5$ such that $2 \leqslant t \leqslant h-1$. In particular, $n_{i}<k-5$ for $i \neq t$. Moreover, $D_{1}^{t-1}$ and $D_{t+1}^{h}$ are tournaments.

Now $D_{2}^{h}$ is a $\left(k-n_{1}\right)$-traceable oriented graph with independence number 2 and $n\left(D_{2}^{h}\right) \geqslant 4 k-12-n_{1}>4\left(k-n_{1}\right)-12$. Hence it follows from our induction hypothesis that $D_{2}^{h}$ is traceable and thus has a Hamilton path with initial vertex $x$ in $D_{2}$.

Now suppose $h \geqslant 4$. Then if $t \geqslant 3$, Theorem 2.3 implies that $\left\langle D_{1}^{2}\right\rangle$ is a tournament. Since $D_{1}$ is hamiltonian or a single vertex and every vertex in $D_{1}$ is adjacent to $x$, it follows that $D$ is traceable. If $t<3$, we consider $D_{1}^{h-1}$ instead of $D_{2}^{h}$ and deduce in a similar manner that $D$ is traceable. We may therefore assume that $h=3$. Thus $D_{1}$ and $D_{3}$ are tournaments, while $D_{2}$ is nonhamiltonian and $n\left(D_{2}\right) \geqslant n-k+5$.

By Theorem 2.1, $D_{1}^{2}$ is traceable, so $D_{2}$ has a Hamilton path $x_{1} \ldots x_{n_{2}}$ such that $x_{1} \in N^{+}\left(D_{1}\right)$. By Lemma 2.4(1), $x_{n_{2}} \notin N^{-}\left(D_{3}\right)$, so it follows from Lemma 2.4(6b) that $d_{D_{2}}^{+}\left(x_{n_{2}}\right) \geqslant n-k+1 \geqslant 3 k-11$, since $n \geqslant 4 k-12$. Let $x_{j}$ be the out-neighbour of $x_{n_{2}}$ such that $x_{n_{2}}$ has exactly $k-3$ out-neighbours in $\left\{x_{1}, \ldots, x_{j}\right\}$. Then $x_{n_{2}}$ has at least $n-2 k+4$ out-neighbours in $\left\{x_{j+1}, \ldots, x_{n_{2}}\right\}$. Hence $n_{2}-2-j \geqslant n-2 k+4$. Since $n_{2} \leqslant n-2$, it follows that $j \leqslant 2 k-8$.

Claim 1. $x_{j-1} \in N^{-}\left(D_{3}\right)$. 
Proof. If $x_{s} \in N^{+}\left(x_{j-1}\right)$ for some $s \geqslant j+1$, then $x_{1} \ldots x_{j-1} x_{s} \ldots x_{n_{2}} x_{j} \ldots x_{s-1}$ is a Hamilton path of $D_{2}$ and hence, by Lemma 2.4(1), $x_{s-1}$ has no out-neighbour in $D_{3}$. But, by Lemma $2.4(5 \mathrm{~b})$, the number of vertices in $D_{2}$ that have no out-neighbours in $D_{3}$ is at most $n_{2}-(n-k+1) \leqslant k-3$, since $n_{2} \leqslant n-2$. Hence $\left|N^{+}\left(x_{j-1}\right) \cap\left\{x_{j+1}, \ldots, x_{n_{2}}\right\}\right| \leqslant k-3$ and hence $N_{D_{2}}^{+}\left(x_{j-1}\right) \leqslant j-1+k-3 \leqslant 3 k-12 \leqslant n-k$, since $j \leqslant 2 k-8$ and $n \geqslant 4 k-12$. Thus $x_{j-1} \in N^{-}\left(D_{3}\right)$ by Lemma $2.4(6 \mathrm{~b})$.

Claim 2. If $x_{i} \in N^{-}\left(x_{1}\right)$, then $i<j$.

Proof. Suppose, to the contrary that $i \geqslant j$. Since $D_{2}$ is nonhamiltonian, $i \neq n_{2}$. If $x_{s} \in N^{+}\left(x_{n_{2}}\right)$, with $s \leqslant j$, then $x_{s-1} \notin N^{-}\left(x_{i+1}\right)$, since otherwise $x_{i+1} \ldots x_{n_{2}} x_{s} \ldots x_{i} x_{1} \ldots$ $x_{s-1} x_{i+1}$ is a Hamilton cycle of $D_{2}$. But $x_{n_{2}}$ has $k-3$ out-neighbours in $\left\{x_{2}, \ldots, x_{j}\right\}$ (by our choice of $j$ ), so at least $k-3$ vertices in $\left\{x_{1}, \ldots, x_{j-1}\right\}$ are not in $N^{-}\left(x_{i+1}\right)$. Hence $\left|N_{D_{2}}^{-}\left(x_{i+1}\right)\right| \leqslant n_{2}-1-(k-3) \leqslant n-k$. Hence, by Lemma 2.4(6a), $x_{i+1} \in N^{+}\left(D_{1}\right)$. But $x_{i+1} \ldots x_{n_{2}} x_{j} \ldots x_{i} x_{1} \ldots x_{j-1}$ is a Hamilton path of $D_{2}$ and, by Claim $1, x_{j-1} \in N^{-}\left(D_{3}\right)$. This contradicts Lemma 2.4(1) and thus proves the claim.

Claim 3. $\left|N^{+}\left(x_{1}\right) \cap\left\{x_{j+1}, \ldots, x_{n_{2}}\right\}\right| \geqslant n-3 k+10$.

Proof. By Lemma 2.4(3), $x_{1}$ has at least $n-k+1$ neighbours in $D_{2}$. But $x_{1}$ has at most $j-1$ neighbours in $\left\{x_{2}, \ldots, x_{j}\right\}$ and, by Claim $2, x_{1}$ has no in-neighbours in $\left\{x_{j+1}, \ldots, x_{n_{2}}\right\}$. Hence, $\left|N^{+}\left(x_{1}\right) \cap\left\{x_{j+1}, \ldots, x_{n_{2}}\right\}\right| \geqslant n-k+1-(j-1) \geqslant n-3 k+10$, since $j \leqslant 2 k-8$.

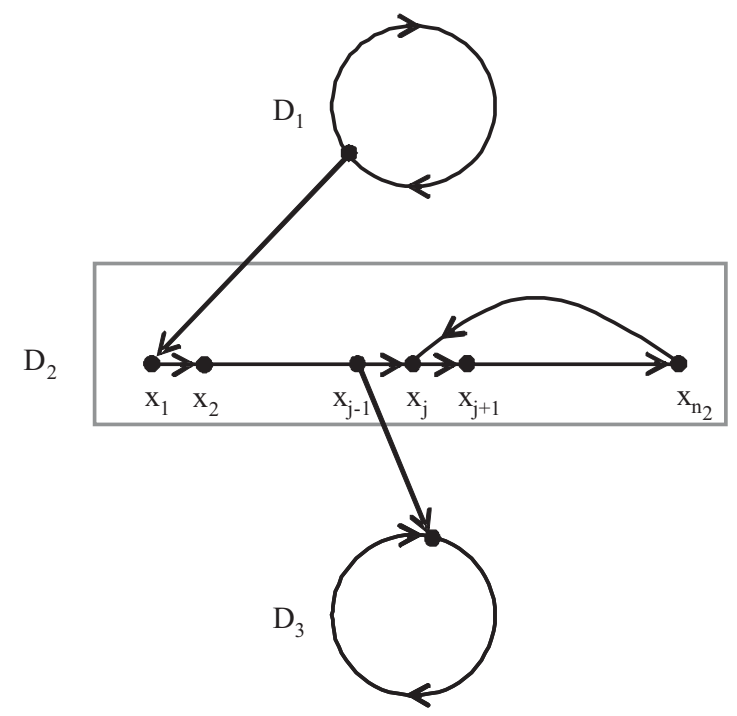

Figure 1: Structure of $D$

Claim 4. $x_{2} \in N^{+}\left(D_{1}\right)$.

Proof. If $x_{i} \in N^{+}\left(x_{1}\right)$ with $i \geqslant j+1$, then $x_{i-1} \notin N^{-}\left(x_{2}\right)$, since otherwise $x_{1} x_{i} \ldots$ $x_{n_{2}} x_{j} \ldots x_{i-1} x_{2} \ldots x_{j-1}$ is a Hamilton path of $D_{2}$ with initial vertex in $N^{+}\left(D_{1}\right)$ and terminal vertex in $N^{-}\left(D_{3}\right)$ (by Claim 1), contradicting Lemma 2.4(1). Thus it follows from 
Claim 3 that $\left|N_{D_{2}}^{-}\left(x_{2}\right)\right| \leqslant n_{2}-1-(n-3 k+10) \leqslant 3 k-13<n-k$ since $n \geqslant 4 k-12$. Hence, by Lemma 2.4(6a), $x_{2} \in N^{+}\left(D_{1}\right)$.

Claim 5. $x_{j-1} \notin N^{-}\left(x_{1}\right)$.

Proof. Since $n \geqslant 4 k-12$, Claim 3 implies that $x_{1}$ has at least $k-2$ out-neighbours in $\left\{x_{j+1}, \ldots, x_{n_{2}}\right\}$. But Lemma 2.4(5b) implies that the number of vertices in $D_{2}$ that are not in $N^{-}\left(D_{3}\right)$ is at most $n_{2}-(n-k+1) \leqslant k-3$. Hence there is an out-neighbour $x_{s}$ of $x_{1}$, with $x_{s} \in\left\{x_{j+1}, \ldots, x_{n_{2}}\right\}$, such that $x_{s-1} \in N^{-}\left(D_{3}\right)$. Now suppose $x_{j-1} \in N^{-}\left(x_{1}\right)$. Then $x_{2} \ldots x_{j-1} x_{1} x_{s} \ldots x_{n_{2}} x_{j} \ldots x_{s-1}$ is a Hamilton path of $D_{2}$. But $x_{2} \in N^{+}\left(D_{1}\right)$ by Claim 4, so this contradicts Lemma 2.4(1).

Claim 6. Let $r$ be the largest integer such that $x_{r} \in N^{-}\left(x_{1}\right)$. Then $x_{r+1} \in N^{+}\left(x_{1}\right)$.

Proof. By Claims 2 and $5, r \leqslant j-2$. If $x_{s} \in N_{D_{2}}^{+}\left(x_{1}\right) \cap\left\{x_{j+1}, \ldots, x_{n_{2}}\right\}$, then $x_{s-1} \notin N^{-}\left(x_{r+1}\right)$, since otherwise $x_{2} \ldots x_{r} x_{1} x_{s} \ldots x_{n_{2}} x_{j} \ldots x_{s-1} x_{r+1} \ldots x_{j-1}$ is a Hamilton path of $D_{2}$ with initial vertex in $N^{+}\left(D_{1}\right)$ and terminal vertex in $N^{-}\left(D_{3}\right)$. Hence, by Claim 3, at least $n-3 k+10$ vertices in $\left\{x_{j}, \ldots, x_{n_{2}-1}\right\}$ are not in $N^{-}\left(x_{r+1}\right)$. By Claim 2 , those vertices are also not in $N^{-}\left(x_{1}\right)$. Hence $\left|N_{D_{2}}^{-}\left(x_{1}\right) \cup N_{D_{2}}^{-}\left(x_{r+1}\right)\right| \leqslant n_{2}-(n-3 k+10) \leqslant$ $3 k-12 \leqslant n-k$. Hence, by Lemma $2.4(4 \mathrm{~b}), x_{1}$ and $x_{r+1}$ are neighbours. But $x_{r+1} \notin N^{-}\left(x_{1}\right)$ by our assumption on $r$, so Claim 6 is proved.

Now, let $\mathcal{P}$ consist of all Hamilton paths in $D_{2}$ whose initial vertices are in $N^{+}\left(D_{1}\right)$. Among all paths in $\mathcal{P}$, choose one that has the largest possible number of vertices between the initial vertex and its last in-neighbour. Denote this path by $Q_{1}=x_{1} \ldots x_{n_{2}}$ and let $x_{r}$ be the last in-neighbour of $x_{1}$ on $Q_{1}$. As $D_{2}$ is nonhamiltonian we have $r<n_{2}$. Let $C$ be the cycle $x_{1} \ldots x_{r} x_{1}$. Then $x_{1}$ has no in-neighbour in $D_{2}-V(C)$. By Claim 6, $x_{1} x_{r+1} \in A\left(D_{2}\right)$ and by Claim $4, x_{2} \in N^{+}\left(D_{1}\right)$. Hence $Q_{2}=x_{2} \ldots x_{r} x_{1} x_{r+1} \ldots x_{n_{2}}$ is also a path in $\mathcal{P}$. Note that $x_{1}$ is the last in-neighbour of $x_{2}$ on $Q_{2}$, by the maximality of $r$. Thus $x_{2}$ has no in-neighbour in $D_{2}-V(C)$. Repeated applications of this procedure show that no vertex on $C$ has an in-neighbour in $D_{2}-V(C)$. This contradicts the fact that $D_{2}$ is strong and thus proves the theorem.

By combining Theorems 1.3 and 3.1, we conclude the following.

Corollary 3.2. $t(k) \leqslant 6 k-20$ for every $k \geqslant 4$.

\section{References}

[1] S.A. van Aardt, A.P. Burger, J.E. Dunbar, M. Frick, J.M. Harris, J.E. Singleton. An iterative approach to the Traceability Conjecture for Oriented Graphs, Electronic J. Comb. 20(1):\#P59, 2013.

[2] S.A. van Aardt, G. Dlamini, J.E. Dunbar, M. Frick, and O.R. Oellermann. The directed path partition conjecture. Discuss. Math. Graph Theory, 25:331-343, 2005.

[3] S.A. van Aardt, J.E. Dunbar, M. Frick, P. Katrenič and M.H. Nielsen, and O.R. Oellermann. Traceability of $k$-traceable oriented graphs. Discrete Math., 310:13251333, 2010. 
[4] S.A. van Aardt, J.E. Dunbar, M. Frick and M.H. Nielsen. Cycles in $k$-traceable oriented graphs. Discrete Math., 311:2085-2094, 2011.

[5] S.A. van Aardt, J.E. Dunbar, M. Frick, M.H. Nielsen, and O.R. Oellermann. A traceability conjecture for oriented graphs. Electron. J. Combin., 15(1):\#R150, 2008.

[6] S. A. van Aardt, A. P. Burger, M. Frick, B. Llano and R. Zuazua. Infinite families of 2-hypohamiltonian/2-hypotraceable oriented graphs. Graphs and Combinatorics, 30(4):783-800, 2014.

[7] S. A. van Aardt, M. Frick, P. Katrenič and M.H. Nielsen. The order of hypotraceable oriented graphs. Discrete Math., 11:1273-1280, 2011.

[8] J. Bang-Jensen, G. Gutin, Digraphs: Theory, Algorithms and Applications, (Second Edition) Springer-Verlag, London, 2009.

[9] J. Bang-Jensen, M.H. Nielsen and A. Yeo. Longest path partitions in generalizations of tournaments. Discrete Math., 306:1830-1839, 2006.

[10] A.P. Burger. Computational results on the traceability of oriented gaphs of small order. Electronic J. Comb. 20(4): \#P23, 2013.

[11] C.C. Chen and P. Manalastas Jr. Every finite strongly connected digraph of stability 2 has a Hamiltonian path. Discrete Math., 44:243-250, 1983.

[12] M. Frick and P. Katrenič. Progress on the traceability conjecture. Discrete Math. and Theor. Comp. Science, 10(3):105-114, 2008.

[13] F. Havet. Stable set meeting every longest path. Discrete Math., 289:169-173, 2004.

[14] J.M. Laborde, C. Payan and N.H. Xuong, Independent sets and longest directed paths in digraphs. In Graphs and other combinatorial topics (Prague, 1982), 173-177 (Teubner-Texte Math., 59, 1983.) 